\title{
Evidence-based medical treatment of peripheral arterial disease: A rapid review
}

\author{
Sze Ling $\underline{\text { Chan }}{ }^{1,2}{ }_{P h D}$, Revvand Rajesh ${ }^{3}$, Tjun Yip $\underline{\text { Tang }}{ }^{2,4}{ }_{F R C S}$
}

\begin{abstract}
Introduction: Peripheral arterial disease (PAD) treatment guidelines recommend the use of statins and antiplatelets in all PAD patients to reduce adverse cardiovascular and limb-related outcomes. In addition, hypertension and diabetes should be treated to reach recommended targets. The aim of this rapid review was to evaluate the level of adherence to evidence-based medical therapy (EBMT) recommended by PAD treatment guidelines in the real-world setting.

Methods: We searched PubMed and Embase using keywords, MeSH and Emtree terms related to the population, exposure and outcomes from their inception to 22 September 2020. We included randomised controlled trials, non-randomised studies, and observational studies reporting adherence to at least 1 of these 4 drug classes: (1) statins, (2) antiplatelets, (3) antihypertensives and (4) antidiabetic drugs. Non-English articles, abstracts, dissertations, animal studies and case reports or series were excluded. A narrative summary of the results was performed.

Results: A total of 42 articles were included in the review. The adherence to lipid-lowering drugs/statins ranged from 23.5 to $92.0 \%$ and antiplatelets from 27.5 to $96.3 \%$. Only 7 and 5 studies reported use of "any anti-hypertensive" and "any anti-diabetic" medications, respectively, and the proportion of the cohort treated were generally close to the proportion with hypertension and/or diabetes. Adherence in studies published in 2016-2020 ranged from 52.4-89.6\% for lipid-lowering drugs and 66.2-96.3\% for antiplatelets.

Conclusion: EBMT adherence in PAD patients was highly variable and a substantial proportion in many settings were undertreated. There was also a notable lack of studies in Asian populations.
\end{abstract}

Ann Acad Med Singap 2021;50:411-24

Keywords: Evidence-practice gap, medication adherence, pharmacoepidemiology

\section{INTRODUCTION}

Peripheral artery disease (PAD) is characterised by debilitating atherosclerotic occlusion of arteries in the lower extremities. ${ }^{1}$ Globally, it was estimated that more than 230 million individuals suffer from PAD in 2015, including about 50 million in Southeast Asia and 74 million in the Western Pacific Region. ${ }^{2}$ The incidence of PAD is increasing significantly across Asia with an advancing age and increasing prevalence of diabetes, which is associated with 2- to 4-fold increase in the incidence of PAD. ${ }^{3}$ Given that Asia has more than 50\% of the diabetes prevalence worldwide, it is estimated that there are several million patients with PAD in Asia, many asymptomatic and undiagnosed. ${ }^{4}$ The severity of PAD ranges from atypical lower-extremity symptoms, intermittent claudication to chronic limb threatening ischaemia (CLTI), which causes rest pain, ulcers or gangrene. ${ }^{5} \mathrm{~A}$ recent systematic review found that CLTI was associated with a 1-year mortality rate of $40 \%$ and 1 -year amputation rates ranged from $15 \%$ to $20 \%{ }^{6}$ Moreover, PAD patients are at an increased risk of cardiovascular morbidity and mortality. ${ }^{1}$ Although endovascular or surgical revascularisation procedures are important facets of PAD management, medical and lifestyle interventions are also essential elements of evidence-based PAD care, both from a perspective of improving cardiovascular mortality as well as limb-related outcomes. ${ }^{1,5}$ Dyslipidaemia

\footnotetext{
${ }^{1}$ Health Services Research Centre, SingHealth, Singapore

${ }^{2}$ Duke-NUS Medical School, Singapore

${ }^{3}$ Lee Kong Chian School of Medicine, Nanyang Technological University, Singapore

${ }^{4}$ Department of Vascular Surgery, Singapore General Hospital, Singapore

Correspondence: Dr Sze Ling Chan, Health Services Research Centre, Singapore Health Services, 20 College Road, Academia Discovery Tower, Level 6, Singapore 169856 .

Email: chan.sze.ling@singhealth.com.sg
} 


\section{CLINICAL IMPACT}

\section{What is New}

- This review provides an update of evidencebased medical therapy (EMBT) used in peripheral arterial disease (PAD) since the last systematic review 12 years ago.

\section{Clinical Implications}

- The review highlights existing gaps in the use of EBMT in PAD patients, given the highly variable level of adherence found for all 4 classes of EBMT.

- There was a lack of Asian studies, pointing to a need for more research on EBMT use in Asian PAD patients.

and hypertension are estimated to nearly double the risk of PAD. ${ }^{1}$ Lifestyle changes and control of risk factors increase the short and long-term patency of angioplasties and surgical bypasses, resulting in a reduction of target lesion revascularisation. ${ }^{1}$

The 2016 American College of Cardiology/American Heart Association (ACC/AHA) Guidelines for the Management of Patients with Peripheral Arterial Disease and 2017 European Society of Cardiology (ESC) Guidelines recommend that PAD patients be treated lifelong with a statin and an antiplatelet drug, aspirin alone (range 75-325mg per day) or clopidogrel alone (75mg per day), to reduce the risk of cardiovascular events and death. ${ }^{1,5}$ All patients should have their serum low-density lipoprotein cholesterol $(\mathrm{LDL}-\mathrm{C})$ reduced to $<1.8 \mathrm{mmol} / \mathrm{L}(<70 \mathrm{mg} / \mathrm{dL})$ or decreased by $>50 \%$ if the initial LDL-C level is between 1.8 and $3.5 \mathrm{mmol} / \mathrm{L}$ ( 70 and $135 \mathrm{mg} / \mathrm{dL}$ ). ${ }^{7}$ Statin therapy has been shown to reduce major extremity amputation by $18 \%$, adverse cardiovascular events by $20 \%$, and all-cause mortality by $19 \% .{ }^{8}$ Furthermore, the use of antiplatelet agents and statins at the time of intervention for PAD patients without known cardiovascular disease has also been associated with better 5-year survival compared to PAD patients receiving no treatment. ${ }^{9}$ In addition, hypertension and diabetes should be treated and controlled. As per guidelines, a target blood pressure $<140 / 90 \mathrm{mmHg}$ is recommended except in patients with diabetes, for whom a diastolic blood pressure $\leq 85 \mathrm{mmHg}$ is considered a safer policy. ${ }^{10}$
Despite these guidelines, there is evidence that PAD patients are undertreated. A systematic review of implementation of recommended secondary prevention in PAD patients found that antiplatelet medication, lipid-lowering agents and antihypertensives were prescribed only in $63 \%, 45 \%$ and $46 \%$ of PAD patients, respectively. ${ }^{11}$ There is also evidence of variation in perioperative antiplatelet and statin usage by procedure and among centres. ${ }^{11}$ This rapid review aims to provide an updated evaluation of the level of adherence to evidence-based medical therapy (EBMT) recommended by PAD treatment guidelines in the real-world setting.

\section{METHODS}

\section{Study selection}

We searched PubMed and Embase using keywords, $\mathrm{MeSH}$ and Emtree terms related to the population, exposure and outcomes (Table 1), from their inception to 22 September 2020. Terms in each element were combined using the Boolean operator "OR" and then results from each element combined using "AND". Filters were applied to restrict language to "English". We included randomised controlled trials, non-randomised studies, and observational studies reporting adherence to at least 1 of these 4 drug classes: (1) statins ("any lipid-lowering drugs" were also included as they are likely to represent overall antilipid treatment including when statins were not suitable), (2) antiplatelets, (3) antihypertensives and (4) antidiabetic drugs. The following types of studies were excluded: (1) full text not available in English, (2) conference abstracts, (3) dissertations, (4) animal studies, or (5) case reports. We also screened reviews but included the primary studies instead of the reviews themselves. Two authors screened the studies identified through the search strategy independently, and conflicts were resolved through discussion with a third reviewer.

There were variable definitions of PAD in the studies screened, with some including diseases of the aorta, carotid and/or renal arteries. For the purposes of this review, we restricted the population to lower extremity PAD (disease affecting the aorta-iliac segments and below). Studies that included other populations but reported the results of interest in the PAD subgroup were included. Among duplicate reports of the same study population or database, we selected reports in this order of priority: (1) those that reported more exposures, (2) larger sample size, and (3) most updated results (later study period or publication date). The Preferred 
Reporting Items for Systematic Reviews and MetaAnalyses (PRISMA) were followed. ${ }^{12}$ This rapid review protocol was also registered in International Prospective Register of Systematic Reviews (PROSPERO) on 12 August 2020.

\section{Data collection}

One reviewer extracted key information and results from each study: first author, publication year, country of origin, setting, study design, study period, population inclusion and exclusion criteria, sample size, exposures, outcome measurement method, outcomes, and information needed for risk of bias assessment. A random $10 \%$ sample was checked by a second reviewer and the compliance rate was $100 \%$. Risk of bias was assessed using the National Heart, Lung and Blood Institute (NHLBI) standardized Quality Assessment Tool by one reviewer and was randomly checked by the second reviewer. ${ }^{13}$

\section{Data analysis}

As the studies were rather diverse in the types of PAD patients included, context and definition of adherence, we performed a narrative summary of the results. For studies that measured adherence trend over several years, we took the value at the latest time period where available. However, for studies that reported adherence at different timepoints relative to a medical encounter for PAD diagnosis, intervention or management (e.g. before admission, after admission and follow-up), the data for each timepoint were retained as this provides a view on the effect of the encounter on quality of treatment. In these cases, the latest time point reported was used for showing the overall trend.

For the main results, we only showed the overall drug classes and the main drug or drug class of choice to simplify data presentation, rather than all specific drug entities or classes. For studies that reported angiotensin-converting enzyme inhibitor (ACEI) and angiotensin receptor blockers (ARBs) separately but not ACEI/ARBs, this was imputed by adding ACEI and ARBs as these drugs were generally not used together. Results for ACEIs alone were also presented in the same category. For antidiabetic medications, only results for "any antidiabetics" and/or metformin (drug of choice) were shown. We also explored the results by continent of origin and publication year to determine if there were regional differences and if adherence has improved over time. Due to the varied nature of the PAD populations included, it was difficult to accurately group them by severity. Comorbidities were also not consistently reported to allow for subgroup analysis by these characteristics. All analyses were conducted in $\mathrm{R}$ version 3.5.1. ${ }^{14}$

\section{RESULTS}

\section{Search results}

The search strategy yielded a total of 1,843 articles from both PubMed and Embase as of 22 September 2020; 42 articles were finally included in this review (Fig. 1). The details of the included studies are shown in Table 2. The studies were published between 2004 and 2020, with almost two-thirds in the last 5 years. Most of the studies originated from the US or Europe, with only 1 paper from Asia. The median sample size was 588 patients (range $72-175,865$ ). The type of PAD populations in the included studies varied from those patients with mild symptoms (only intermittent claudication) to those with rest pain/tissue loss (CLTI). Thirty-four studies $(81 \%)$ were rated as "good" quality while the remaining 8 were "fair", according to the NHLBI quality assessment tool.

\section{Overall adherence to EBMT}

Of the 42 included studies, 41 (97.6\%), 31 (73.8\%), 25 $(59.5 \%)$ and $8(19.0 \%)$ studies reported adherence on lipid-lowering drugs, antiplatelets, antihypertensives and antidiabetic drugs, respectively. However, among the 25 studies that reported antihypertensives, only 7 (28/0\%) reported use of "any antihypertensives", $15,16,31,35,44,45,52$ and among the 8 studies that reported antidiabetic drugs, only $5(62.5 \%)$ reported use of "any antidiabetics". $15,16,39,44,52$ The rest reported specific drugs or classes of antihypertensives/antidiabetics separately, and it was not possible to infer the proportion of the cohort who received pharmacological treatment as patients may be on multiple agents.

There was substantial variability in the level of adherence to all 4 classes of drugs (Fig. 2). The adherence to lipid-lowering drugs or statins ranged from 23.5 to $92.0 \%$ and antiplatelets from 27.5 to $96.3 \%$. There was a strong correlation between adherence to lipid-lowering drugs and antiplatelets. Studies that had low adherence to lipid-lowering drugs also tended to have low antiplatelet adherence, and vice versa (Pearson's $r=0.81, P<0.0001$ ).

The adherence to antihypertensives and antidiabetics were also highly variable (Figure 2). These values reflect the proportion of the entire PAD cohort treated 
Table 1. Search terms

\begin{tabular}{|c|c|c|c|}
\hline Element & Plain text & MeSH terms (PubMed) & Emtree terms (EMBASE) \\
\hline Population & $\begin{array}{l}\text { "peripheral arterial disease" OR "peripheral } \\
\text { artery disease" OR "chronic limb ischemia" } \\
\text { OR "chronic limb threatening ischemia" } \\
\text { OR (limb AND ischemia) OR (limb AND } \\
\text { atherosclerosis) }\end{array}$ & "peripheral arterial disease" & "peripheral occlusive artery disease" \\
\hline Exposure & $\begin{array}{l}\text { "medical therapy" OR "medical treatment" } \\
\text { OR } \\
\text { guideline* OR medication* OR drug* OR } \\
\text { aspirin OR clopidogrel OR antiplatelet* OR } \\
\text { anticoagulant* OR statin* OR } \\
\text { antihypertensive OR antidiabetic OR } \\
\text { antihyperglycemic OR hypoglycemic OR } \\
\text { "antithrombotic agent" }\end{array}$ & $\begin{array}{l}\text { "evidence-based practice" OR aspirin } \\
\text { OR clopidogrel OR "platelet aggregation } \\
\text { inhibitors" OR anticoagulants OR } \\
\text { "hydroxymethylglutaryl-CoA reductase } \\
\text { inhibitors" OR "antihypertensive agents" } \\
\text { OR "hypoglycemic agents" }\end{array}$ & $\begin{array}{l}\text { "drug therapy" OR "evidence-based } \\
\text { practice" OR "acetylsalicylic acid" OR } \\
\text { clopidogrel OR "antithrombocytic } \\
\text { agent" OR "anticoagulant agent" OR } \\
\text { "hydroxymethylglutaryl coenzyme } \\
\text { A reductase inhibitor" OR } \\
\text { "antihypertensive agent" OR } \\
\text { "antidiabetic agent" }\end{array}$ \\
\hline Outcome & adherence OR compliance & $\begin{array}{l}\text { "medication adherence" OR "guideline } \\
\text { adherence" OR "treatment adherence and } \\
\text { compliance" }\end{array}$ & "medication compliance" \\
\hline
\end{tabular}

*: wildcard search symbol

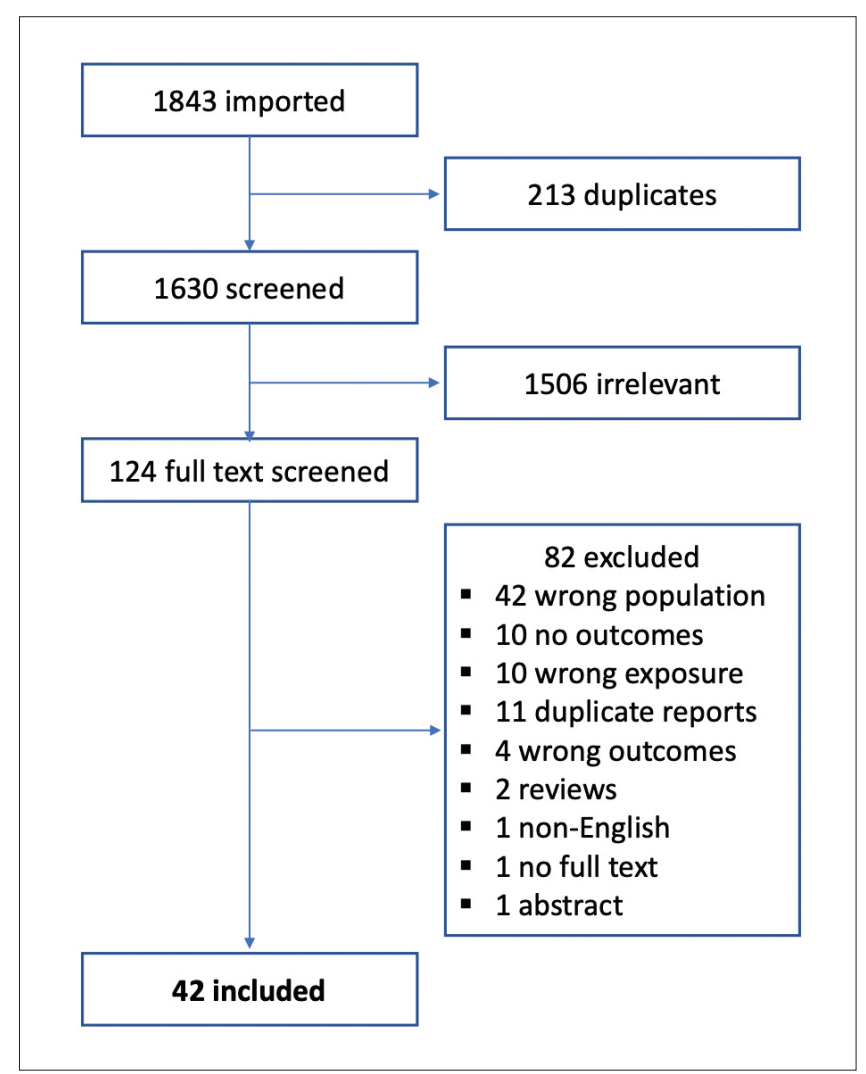

Fig. 1. Preferred Reporting Items for Systematic Reviews and MetaAnalyses (PRISMA) diagram.

with these drugs, so the proportion of those with hypertension and diabetes were also indicated where available to provide more context for the results (Fig. 2, light grey squares). Several studies have very high or $100 \%$ adherence relative to the proportion of the cohort who had the corresponding comorbidities (hypertension or diabetes). ${ }^{15,16,31,45,46,52,54}$ For the other studies with seemingly suboptimal antihypertensive adherence, only ACEI/ARBs were represented and/or the proportion of the cohort with hypertension was unknown. Similarly, for antidiabetics, either only metformin was represented or the proportion of diabetics was unknown. ${ }^{31,39}$

\section{Adherence to EBMT by continent and publication year}

European and North American studies had similarly wide distributions in adherence to both lipid-lowering drugs (31.3-92.0\% and $23.5-85.3 \%$, respectively) and antiplatelets (27.5-92.9\% and $44.5-91.3 \%$, respectively). International studies tended to have higher adherence (range $64.2-89.6 \%$ for lipid-lowering drugs and $81.7-89.6 \%$ for antiplatelets) (Fig. 3 panel A).

When grouped by publication year, there was a trend of increasing adherence with newer studies. Adherence in studies published in 2016-2020 ranged from $52.4-89.6 \%$ for lipid-lowering drugs and $66.2-96.3 \%$ for antiplatelets (Fig. 3 panel B).

\section{Adherence at different timepoints around a medical encounter}

In general, adherence to all classes of medications improved immediately after discharge from an admission or consultation for peripheral arterial disease diagnosis, intervention or management, when compared to before the encounter (Fig. 4). However, in studies that continue to follow up further, adherence tends to drop slightly 


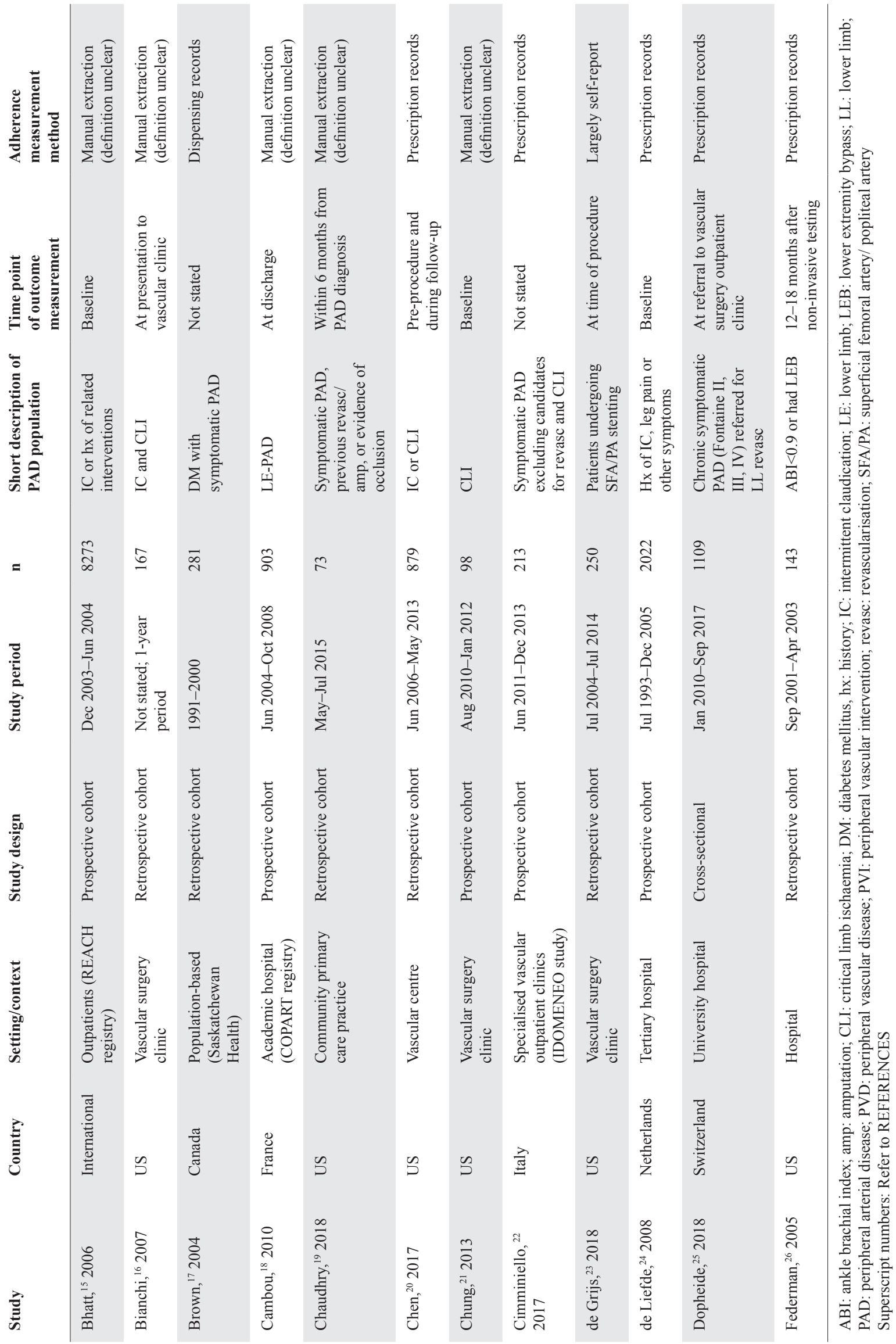




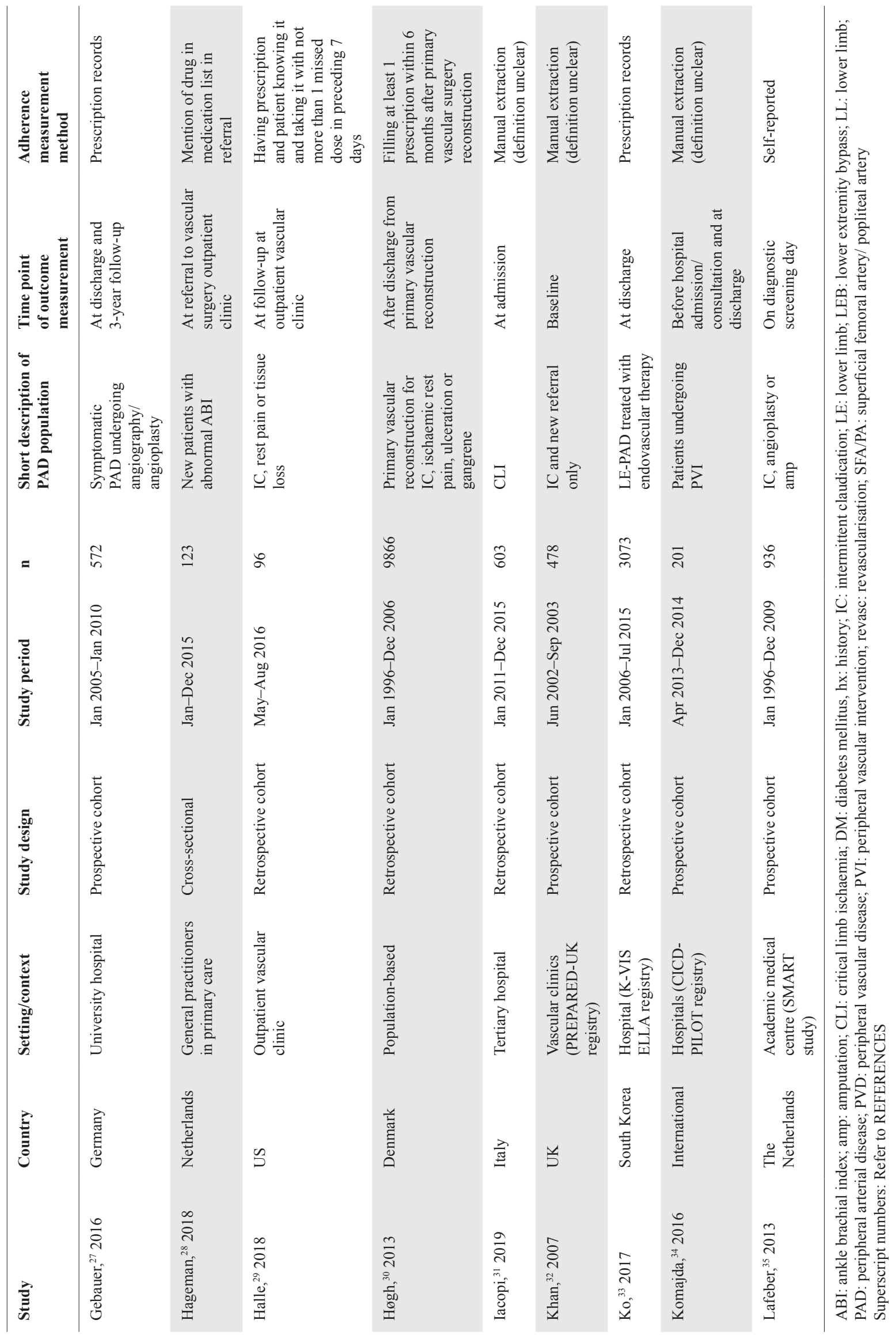




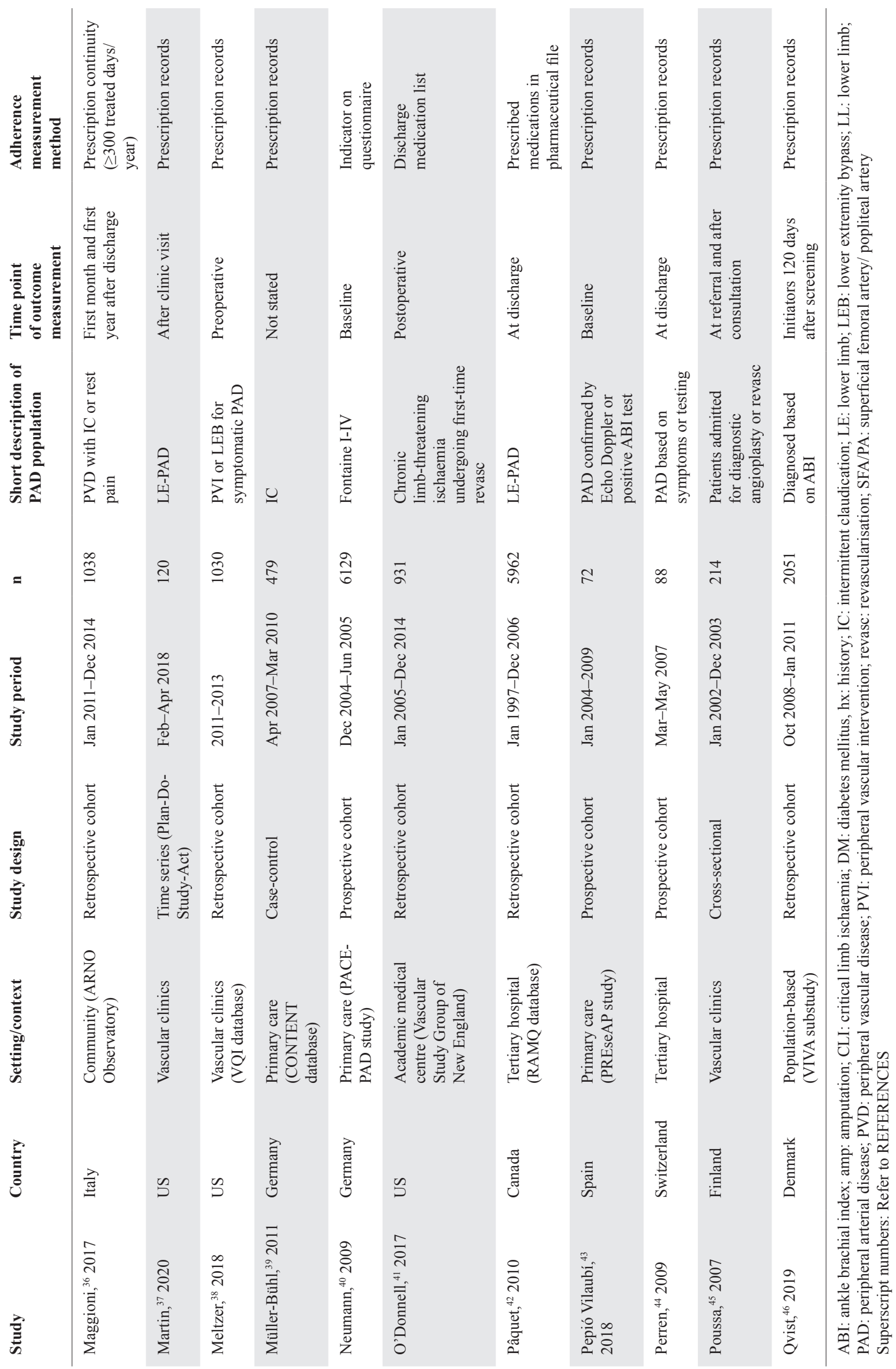




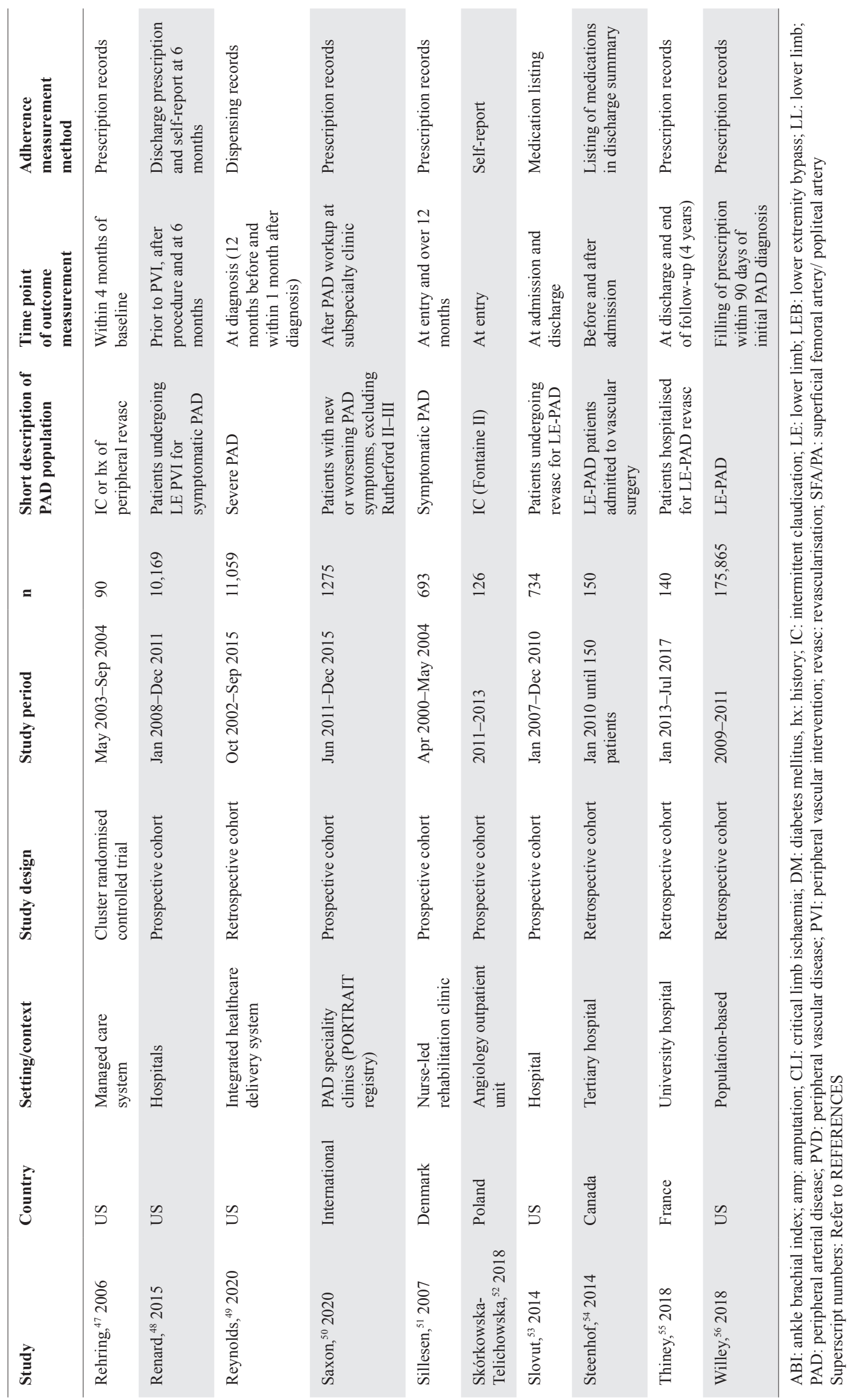




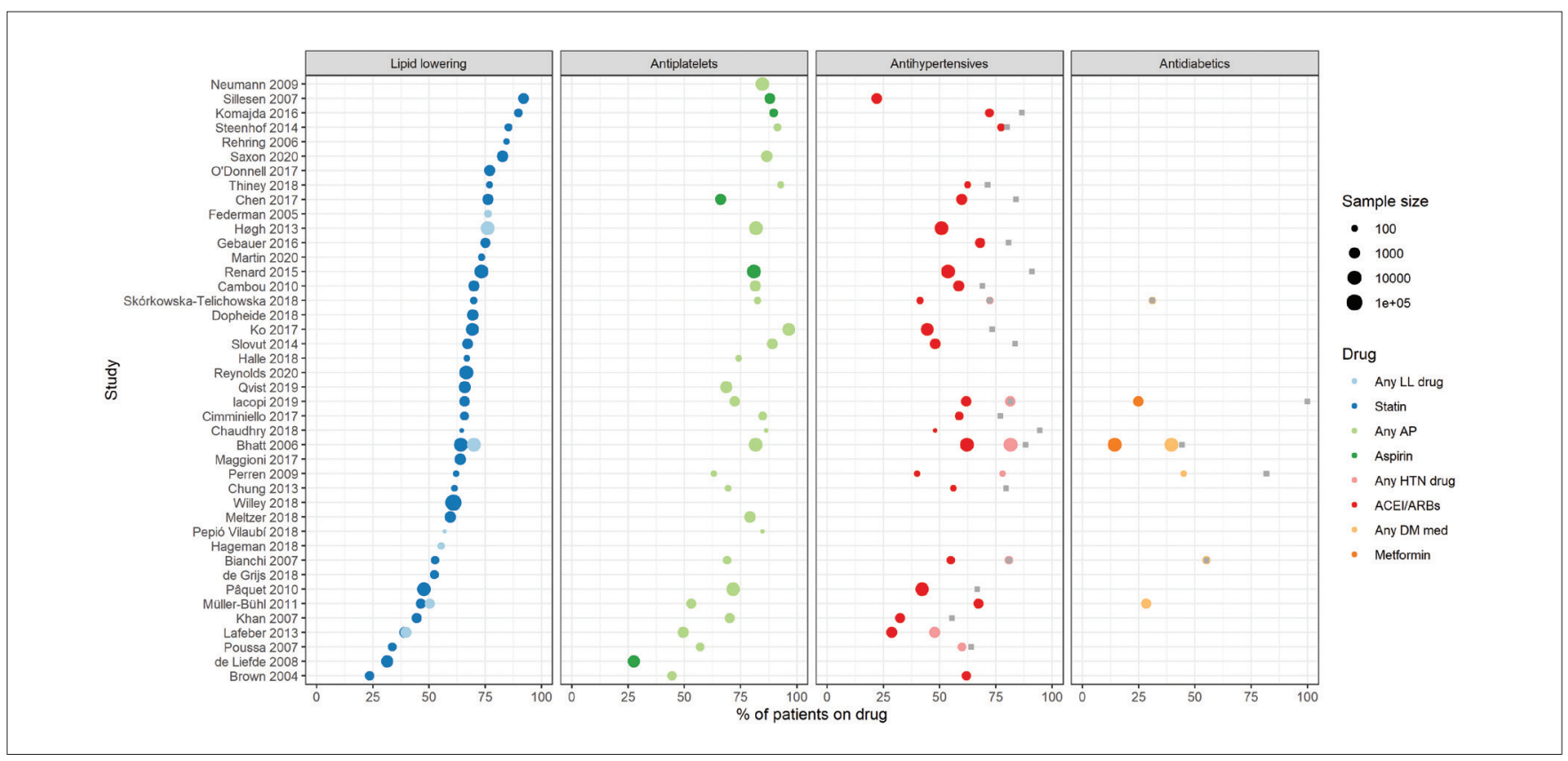

Fig. 2. Adherence to lipid-lowering drugs, antiplatelets, antihypertensives and antidiabetic medications.

For studies that reported adherence at different time points, the latest one is presented here. For antihypertensives and antidiabetic medications, the results reflect the percentage of the entire peripheral artery disease cohort taking those drugs. The light grey squares in the antihypertensives and diabetes mellitus medications panels represent the percentage of the cohort that have hypertension and diabetes, respectively.

LL: lipid lowering; AP: antiplatelets; HTN: hypertension; ACEI: angiotensin converting enzyme inhibitor; ARB: angiotensin receptor blocker; DM: diabetes mellitus

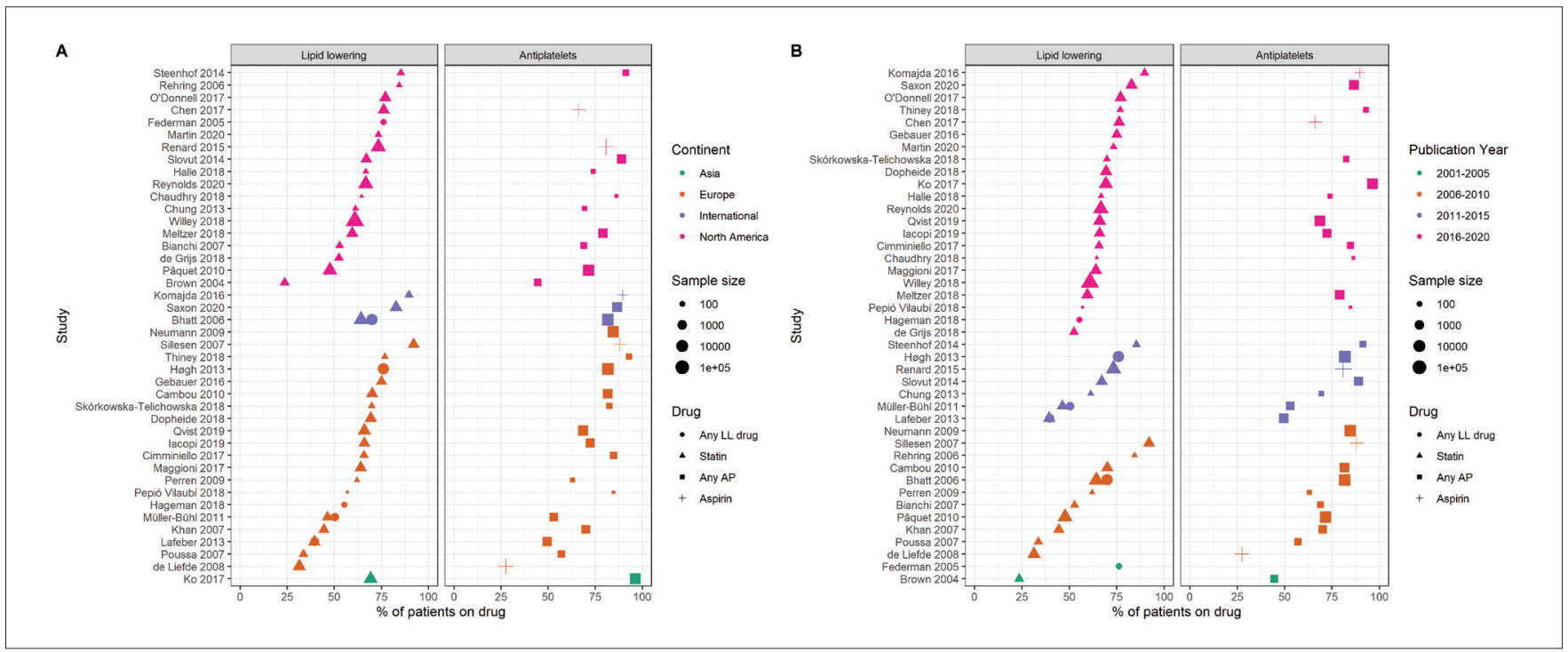

Fig. 3. Adherence to lipid-lowering drugs and antiplatelets by continent and publication year.

For studies that reported adherence at different time-points, the latest one is presented here. Panel A shows similarly wide distributions in adherence to both drug classes in Europe and North America, whereas international studies tended to have higher adherence. Panel B shows a general increasing trend in adherence to both drug classes over time.

LL: lipid lowering; AP: antiplatelets

(absolute decrease ranging from 0.4 to $11.0 \%$ ),,$^{27,48,55}$ with an exception in Maggioni et al., where an absolute increase of $26.5 \%$ might have been contributed by the high rate of readmissions within the first year. However, over a longer follow-up of 3 years, adherence to statins (in the entire cohort including patients with acute coronary syndrome and cardiovascular disease) did drop from 59.9 to $48.4 \%{ }^{36}$ 


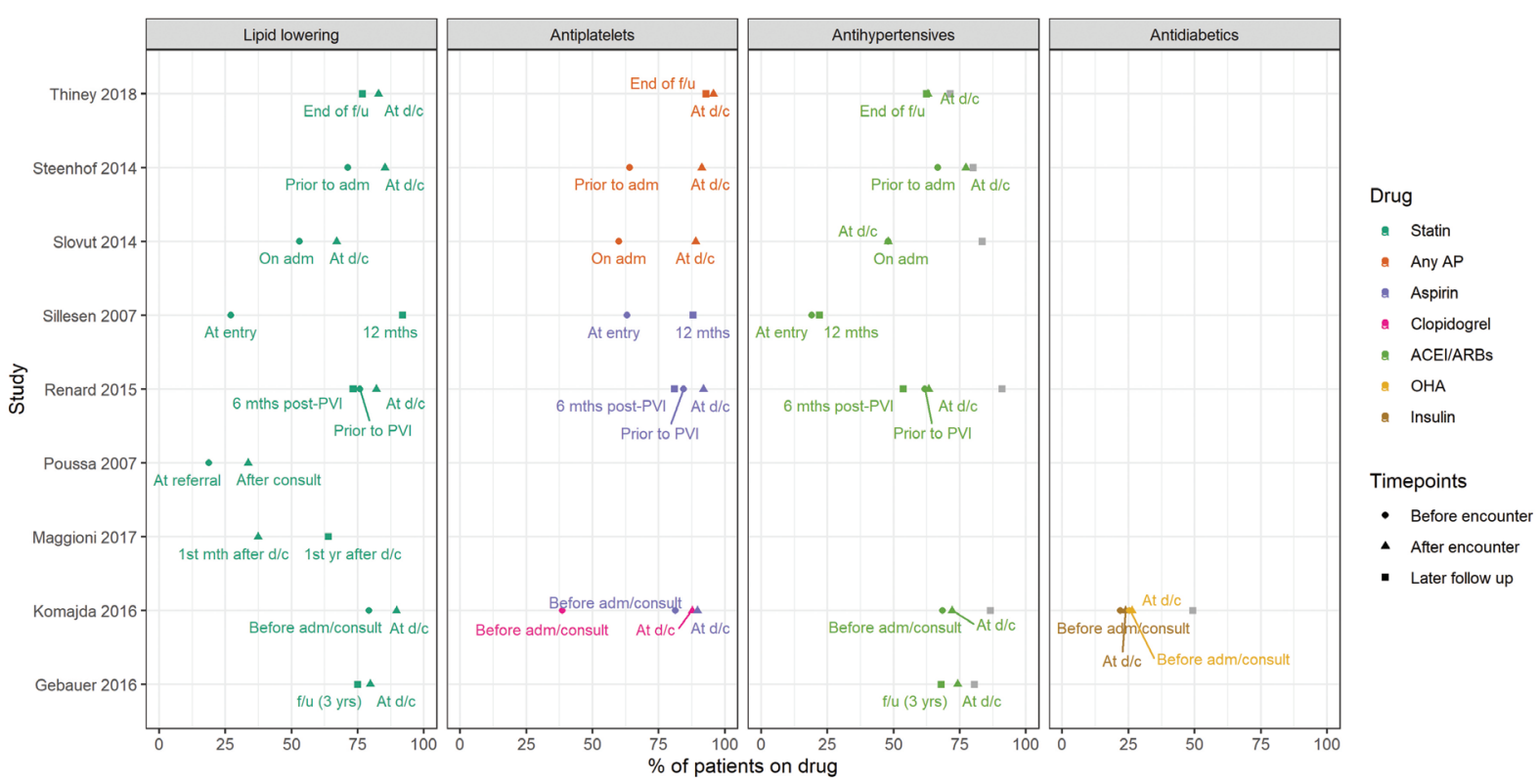

Fig. 4. Effect of healthcare encounter for peripheral artery disease (PAD) on drug adherence.

The adherence at different time-points relative to a medical encounter for PAD is shown. The medical encounter is usually a consultation or an admission for a PAD-related intervention. Generally, adherence is improved after the encounter and falls slightly upon further follow-up. For antihypertensives and antidiabetic medications, the results reflect the percentage of the entire PAD cohort taking those drugs. The light grey squares in the antihypertensives and diabetes mellitus medications panels represent the percentage of the cohort that have hypertension and diabetes, respectively.

f/u: follow-up; d/c: discharge; adm: admission; PVI: peripheral vascular intervention; AP: antiplatelets; ACEI: angiotensin converting enzyme inhibitor; ARB: angiotensin receptor blocker; OHA: oral hypoglycaemic agent

\section{DISCUSSION}

Twelve years after the last systematic review on the use of EBMT in PAD patients, the situation remains sadly the same. ${ }^{11}$ The main findings of this review showed that medical therapy adherence was highly variable, and undertreatment with EBMT in PAD patients was common. Another striking finding was the lack of Asian studies. We found only one Korean study, ${ }^{33}$ highlighting a gap in the literature for Asian PAD patients in general. This was also the same as the previous systematic review, which had one study from China. ${ }^{11}$ The Korean study included 3,434 patients treated for intermittent claudication or CLTI from 2006 to 2015 at 31 hospitals across Korea, and found prescription rates at discharge to be relatively high for any antiplatelet drug $(96.3 \%)$ but moderate for statins (69.2\%). ${ }^{33}$ However, in the Chinese study from the previous systematic review, PAD patients with another cardiovascular risk factor had much lower prescription rates for antiplatelets $(72.4 \%)$ and statins $(41.9 \%) .{ }^{57}$ The variation mirrors that seen in North America and Europe, suggesting that there may be local contextual factors affecting the implementation of PAD guidelines.

We used the 2016 ACC/AHA and 2017 ESC guidelines for management of PAD patients as the reference for best medical therapy. ${ }^{1}$ Both guidelines, which originated from different continents, as well as the global vascular guidelines for CLTI patients, ${ }^{58}$ agree on the universal treatment of PAD patients with statins and antiplatelets, and control of hypertension and diabetes in those who have them. The ESC guidelines recommend ACEI/ $\mathrm{ARBs}$ as first line therapy for hypertension in PAD patients, while metformin was recommended as the hypoglycaemic agent of choice in the global vascular guidelines for CLTI patients. ${ }^{5,58}$ Unlike the previous systematic review by Flu et al., we did not focus on heart rate lowering medications because this was not a class of drug specifically recommended in the guidelines. ${ }^{1,5,11}$ In fact, beta blockers had been thought to worsen PAD symptoms but this has been revised and beta blockers are now considered acceptable treatments in recent guidelines. ${ }^{1}$ We also excluded exercise therapy and smoking cessation to focus on EBMT.

The evidence supporting these recommendations have been present for a long time and the same recommendations have been made as early as in $1999 .{ }^{59}$ However, there are gaps in knowledge and action on the recommended treatment targets for vascular surgeons..$^{60}$ One of several potential reasons is low provider comfort levels in treating PAD patients. ${ }^{45}$ The 
previous systematic review by Flu et al. also summarises possible factors for suboptimal implementation of recommended secondary prevention in PAD patients. The authors grouped them into patient-related factors (understanding of disease, compliance, polypharmacy), physician-related factors (underdiagnosis of PAD, lack of knowledge of risk factor modification, lack of time and reimbursement) and healthcare-related factors (responsibility is spread out). ${ }^{11}$ A systematic meta-review of the barriers and facilitators to implementation of clinical practice guidelines echoed many of these factors but included factors in the guideline, and political and social contexts. ${ }^{61}$ In particular, consistent leadership, which provides clear objectives of care and rallies together care providers from difference disciplines, is a key facilitator. ${ }^{61}$

The large variation we saw in the level of adherence to EBMT is likely due to varying efforts in the implementation. We observed a trend of increasing adherence with time, possibly due to the increasing recognition that focused efforts are needed for understanding the implementation factors and designing strategies to overcome the barriers to implementing clinical practice guidelines in recent years. ${ }^{61}$ In particular, collaborative care between vascular surgeons, primary physicians and internists, is important and effective in bringing about better EBMT adherence and thus long-term outcomes, given that the responsibility of instituting EBMT is unclear otherwise. ${ }^{60}$ Suboptimal adherence to EBMT is associated with higher risk of major amputation and death. ${ }^{21}$ Statin use is associated with lower major adverse cardiovascular events and mortality. ${ }^{56}$ It is therefore important that more research efforts are directed towards understanding and improving implementation of the PAD guidelines into practice.

It was difficult to ascertain the quality of hypertension and diabetes treatment due to the variability in the type of drugs or drug classes that were reported. For hypertension, ACEI/ARBs are the drugs of choice according to the ESC guidelines, but treatment with any antihypertensive would have been considered adherent. ${ }^{5}$ Among the 6 studies that reported use of "any antihypertensive", the level of use was quite close to the proportion with hypertension, suggesting that majority of hypertensive patients were being treated. ${ }^{15,16,31,35,44,45}$ Comparing the level of ACEI/ARBs use with "any antihypertensive", it appears that ACEI/ARBs were not always the drug of choice. ${ }^{15,31,35}$ In one study though, ACEI/ARBs use was very close to the hypertensive proportion. ${ }^{54}$ For diabetes, treatment was $100 \%$ in 2 studies $^{16,52}$ but less than ideal in the study by Perren et al. ${ }^{44}$ For these 2 conditions, we reported the level of use in the entire cohort and the proportion with the conditions for context, instead of assuming that antihypertensives and antidiabetic drugs are only used in patients with hypertension and diabetes, respectively, as it may not be necessarily the case.

Our protocol initially included anticoagulants as an exposure, as guidelines recommend that they should not be used for prevention of atherosclerosis. . $^{1,5,58}$ However, in the course of the review it was difficult to establish if there were other indications for anticoagulants, so we decided to exclude this exposure. Some studies reported use of "antiplatelets and/or anticoagulants" but again it was not possible to establish if patients had good justifications to be given anticoagulants instead of antiplatelets. The recent COMPASS and VOYAGER trials showed that the combination of low-dose rivaroxaban and aspirin significantly reduced the incidence of acute or chronic limb ischaemia and its related complications (amputations and death) compared to aspirin alone, suggesting a potential role for rivaroxaban in the management of PAD patients. However, the risk of major bleeding is higher with the combination of an antiplatelet and anticoagulant, and the decision to use requires an individualised assessment of the risks and benefits. ${ }^{62}$

The 9 studies that reported adherence at multiple timepoints relative to a medical encounter for PAD diagnosis or treatment suggested that these encounters were opportunities to review and institute EBMT in patients who have not been receiving them (Fig. 4). In one setting where CLTI patients were managed in a nurse-led PAD rehabilitation clinic, the statin adherence rate increased dramatically from 27 to $92 \%$ after a year with 5 visits. ${ }^{51}$ This was a successful example of active implementation of EBMT in practice, through collaborative management of the patients, regular monitoring and feedback to patients and their family and/or family practitioners on their performance on risk factor targets using printouts. ${ }^{51}$ Other care settings for PAD patients will need to understand their context, barriers and facilitators to find a strategy that works for improving EBMT adherence rates.

There are several limitations in our study. Firstly, there was some variability in the outcome definitions in the studies. Most of the studies measure adherence by whether patients were prescribed the drugs, and this was ascertained by dispensing records, documentation in various sources, and even self-reporting. This therefore represents a mixture of physician's and patient's adherence. Secondly, the results may have been an underestimate of physicians' adherence to 
EBMT prescription. There could be legitimate reasons for withholding certain treatments or drug substitution (e.g. other lipid-lowering agents instead of a statin), but the reasons were not captured in most studies. Also, if dispensing records were used, the non-adherence could be on the patients' part. Despite these issues, the results still provided useful indications of EBMT exposure in PAD patients. Thirdly, the reported adherence levels do not reflect actual patient adherence. Having a prescription or dispensing record does not necessarily mean drug administration. However, this is an inherent limitation of large scale pharmacoepidemiological studies. Fourthly, by including only articles published in English, we might have missed some studies. Lastly, our review did not include most of the primary studies in the previous systematic review by Flu et al. likely due to the slightly different search terms used. ${ }^{11}$ However, we found very similar results so this was unlikely to have affected our conclusions.

\section{CONCLUSION}

In conclusion, EBMT adherence in PAD patients is highly variable and a substantial proportion in many settings are undertreated. Our results also point to research gaps in 2 areas. Firstly, more Asian studies of EBMT adherence are needed. Secondly, for settings with less-than-ideal EBMT adherence, implementation studies on strategies to improve adherence to EBMT are the logical next steps.

\section{REFERENCES}

1. Writing Committee Members, Gerhard-Herman MD, Gornik HL, et al. $2016 \mathrm{AHA} / \mathrm{ACC}$ guideline on the management of patients with lower extremity peripheral artery disease: executive summary. Vasc Med 2017;22:NP1-43.

2. Song P, Rudan D, Zhu Y, et al. Global, regional, and national prevalence and risk factors for peripheral artery disease in 2015: an updated systematic review and analysis. Lancet Glob Health 2019;7:e1020-30.

3. Beckman JA, Creager MA, Libby P. Diabetes and atherosclerosis: epidemiology, pathophysiology, and management. JAMA 2002; 287:2570-81.

4. Ogurtsova K, da Rocha Fernandes JD, Huang Y, et al. IDF Diabetes Atlas: global estimates for the prevalence of diabetes for 2015 and 2040. Diabetes Res Clin Pract 2017;128:40-50.

5. Aboyans V, Ricco JB, Bartelink MEL, et al. 2017 ESC guidelines on the diagnosis and treatment of peripheral arterial diseases, in collaboration with the European Society for Vascular Surgery (ESVS). Eur Heart J 2018;39:763-816.

6. Duff S, Mafilios MS, Bhounsule P, et al. The burden of critical limb ischemia: a review of recent literature. Vasc Health Risk Manag 2019;15:187-208.

7. Piepoli MF, Hoes AW, Agewall S, et al. 2016 European Guidelines on cardiovascular disease prevention in clinical practice. Atherosclerosis 2016;252:207-74.
8. Ramos R, Garcia-Gil M, Comas-Cufi M, et al. Statins for prevention of cardiovascular events in a low-risk population with low ankle brachial index. J Am Coll Cardiol 2016;67:630-40.

9. Pande RL, Perlstein TS, Beckman JA, et al. Secondary prevention and mortality in peripheral artery disease: National Health and Nutrition Examination Study, 1999 to 2004. Circulation 2011; 124:17-23.

10. Mancia G, Fagard R, Narkiewicz K, et al. 2013 ESH/ESC Guidelines for the management of arterial hypertension. J Hypertens 2013; 31:1281-357.

11. Flu HC, Tamsma JT, Lindeman JH, et al. A systematic review of implementation of established recommended secondary prevention measures in patients with PAOD. Eur J Vasc Endovasc Surg 2010;39:70-86.

12. McInnes MDF, Moher D, Thombs BD, et a 1. Preferred reporting items for a systematic review and meta-analysis of diagnostic test accuracy studies: the PRISMA-DTA statement. JAMA 2018;319:388-96.

13. National Heart, Lung, and Blood Institute. Study quality assessment tools. Available at: https://www.nhlbi.nih.gov/health-topics/studyquality-assessment-tools. Accessed on 16 Jul 2020.

14. R Core Team. R: A Language and Environment for Statistical Computing, 3.5.1 Ed. Vienna: R Foundation for Statistical Computing; 2018.

15. Bhatt DL, Steg PG, Ohman EM, et al. International prevalence, recognition, and treatment of cardiovascular risk factors in outpatients with atherothrombosis. JAMA 2006;295:180-9.

16. Bianchi C, Montalvo V, Ou HW, et al. Pharmacologic risk factor treatment of peripheral arterial disease is lacking and requires vascular surgeon participation. Ann Vasc Surg 2007;21:163-6.

17. Brown LC, Johnson JA, Majumdar SR, et al. Evidence of suboptimal management of cardiovascular risk in patients with type 2 diabetes mellitus and symptomatic atherosclerosis. CMAJ 2004;171:1189-92.

18. Cambou JP, Aboyans V, Constans J, et al. Characteristics and outcome of patients hospitalised for lower extremity peripheral artery disease in France: the COPART Registry. Eur J Vasc Endovasc Surg 2010;39:577-85.

19. Chaudhry AP, Afzal N, Abidian MM, et al. Innovative informatics approaches for peripheral artery disease: current state and provider survey of strategies for improving guideline-based care. Mayo Clin Proc Innov Qual Outcomes 2018;2:129-36.

20. Chen DC, Singh GD, Armstrong EJ, et al. Long-term comparative outcomes of patients with peripheral artery disease with and without concomitant coronary artery disease. Am J Cardiol 2017;119:1146-52.

21. Chung J, Timaran DA, Modrall JG, et al. Optimal medical therapy predicts amputation-free survival in chronic critical limb ischemia. J Vasc Surg 2013;58:972-80.

22. Cimminiello C, Polo Friz H, Marano G, et al. Patterns and determinants of use of pharmacological therapies for intermittent claudication in PAD outpatients: results of the IDOMENEO study. Int Angiol 2017;36:254-60.

23. de Grijs D, Teixeira P, Katz S. The association of statin therapy with the primary patency of femoral and popliteal artery stents. J Vasc Surg 2018;67:1472-9.

24. de Liefde II, Hoeks SE, van Gestel YR, et al. Prognostic value of hypotensive blood pressure response during single-stage exercise test on long-term outcome in patients with known or suspected peripheral arterial disease. Coron Artery Dis 2008;19:603-7. 
25. Dopheide JF, Papac L, Schindewolf M, et al. Poor attainment of lipid targets in patients with symptomatic peripheral artery disease. J Clin Lipidol 2018;12:711-7.

26. Federman DG, Ranani DC, Kirsner RS, et al. Lipid-lowering therapy in patients with peripheral arterial disease: are guidelines being met? Mayo Clin Proc 2005;80:494-8.

27. Gebauer K, Engelbertz C, Malyar NM, et al. Long-term mortality after invasive angiography and endovascular revascularization in patients with PAD having chronic kidney disease. Angiology 2016;67:556-64.

28. Hageman D, Pesser N, Gommans LNM, et al. Limited adherence to peripheral arterial disease guidelines and suboptimal ankle brachial index reliability in Dutch primary care. Eur J Vasc Endovasc Surg 2018;55:867-73.

29. Halle TR, Benarroch-Gampel J, Teodorescu VJ, et al. Surgical intervention for peripheral artery disease does not improve patient compliance with recommended medical therapy. Ann Vasc Surg 2018;46:104-11.

30. Høgh A, Lindholt JS, Nielsen H, et al. Secondary medical prevention after primary vascular surgery between 1996 and 2006: a shift towards more evidence-based treatment. Eur J Prev Cardiol 2013; 20:763-70

31. Iacopi E, Coppelli A, Riitano N, et al. Adherence to guideline recommended medical therapies in type 2 diabetic patients with chronic critical limb ischemia. Diabetes Res Clin Pract 2019; 158:107898.

32. Khan S, Flather M, Mister R, et al. Characteristics and treatments of patients with peripheral arterial disease referred to UK vascular clinics: results of a prospective registry. Eur J Vasc Endovasc Surg 2007;33:442-50.

33. Ko YG, Ahn CM, Min PK, et al. Baseline characteristics of a retrospective patient cohort in the Korean Vascular Intervention Society Endovascular Therapy in Lower Limb Artery Diseases (K-VIS ELLA) Registry. Korean Circ J 2017;47:469-76.

34. Komajda M, Weidinger F, Kerneis M, et al. EURObservational Research Programme: the Chronic Ischaemic Cardiovascular Disease Registry: Pilot phase (CICD-PILOT). Eur Heart J 2016;37:152-60.

35. Lafeber M, Grobbee DE, Spiering W, et al. The combined use of aspirin, a statin, and blood pressure-lowering agents (polypill components) in clinical practice in patients with vascular diseases or type 2 diabetes mellitus. Eur J Prev Cardiol 2013;20:771-8.

36. Maggioni AP, Calabria S, Rossi E, et al. Use of lipid lowering drugs in patients at very high risk of cardiovascular events: an analysis on nearly 3,000,000 Italian subjects of the ARNO Observatory. Int J Cardiol 2017;246:62-7.

37. Martin C, Phillips R, Johnson C. Improving provider awareness to statin prescribing guidelines: a quality improvement project. J Vasc Nurs 2020;38:25-8.

38. Meltzer AJ, Sedrakyan A, Connolly PH, et al. Risk factors for suboptimal utilization of statins and antiplatelet therapy in patients undergoing revascularization for symptomatic peripheral arterial disease. Ann Vasc Surg 2018;46:234-40.

39. Müller-Bühl U, Laux G, Szecsenyi J. Secondary pharmacotherapeutic prevention among German primary care patients with peripheral arterial disease. Int J Vasc Med 2011;2011:316496

40. Neumann A, Jahn R, Diehm C, et al. Presentation and medical management of peripheral arterial disease in general practice: rationale, aims, design and baseline results of the PACE-PAD Study. J Public Health 2009; 17:127-35.
41. O'Donnell TFX, Deery SE, Darling JD, et al. Adherence to lipid management guidelines is associated with lower mortality and major adverse limb events in patients undergoing revascularization for chronic limb-threatening ischemia. J Vasc Surg 2017;66:572-8.

42. Paquet M, Pilon D, Tetrault JP, et al. Protective vascular treatment of patients with peripheral arterial disease: guideline adherence according to year, age and gender. Can J Public Health 2010; 101:96-100.

43. Pepió Vilaubí JM, Orozco-Beltrán D, Gonçalves AQ, et al. Adherence to European clinical practice guidelines for secondary prevention of cardiovascular disease: a cohort study. Int J Environ Res Public Health 2018;15:1233

44. Perren A, Cerutti B, Lazzaro M, et al. Comparison of in-hospital secondary prevention for different vascular diseases. Eur J Intern Med 2009;20:631-5.

45. Poussa H, Strandberg TE, Tikkanen I, et al. Diagnosis and treatment of dyslipidemia are neglected in patients with peripheral artery disease. Scand Cardiovasc J 2007;41:138-41.

46. Qvist I, Søgaard R, Lindholt JS, et al. Adherence to prescribed drugs among 65-74 year old men diagnosed with abdominal aortic aneurysm or peripheral arterial disease in a screening trial: a VIVA substudy. Eur J Vasc Endovasc Surg 2019;57:442-50.

47. Rehring TF, Stolcpart RS, Sandhoff BG, et al. Effect of a clinical pharmacy service on lipid control in patients with peripheral arterial disease. J Vasc Surg 2006;43:1205-10.

48. Renard BM, Seth M, Share D, et al. If not now, when? Prescription of evidence-based medical therapy prior to hospital discharge increases utilization at 6 months in patients with symptomatic peripheral artery disease. Vasc Med 2015;20:544-50.

49. Reynolds K, Mues KE, Harrison TN, et al. Trends in statin utilization among adults with severe peripheral artery disease including critical limb ischemia in an integrated healthcare delivery system. Vasc Med 2020;25:3-12

50. Saxon JT, Safley DM, Mena-Hurtado C, et al. Adherence to guideline-recommended therapy-including supervised exercise therapy referral-across peripheral artery disease specialty clinics: insights from the international PORTRAIT registry. J Am Heart Assoc 2020;9:e012541.

51. Sillesen H, Madelung S, Eldrup N, et al. Organising a nurse-driven PAD rehabilitation clinic within the vascular surgical department: what is required and are treatment goals reached-a prospective study? Eur J Vasc Endovasc Surg 2007;33:26-32.

52. Skórkowska-Telichowska K, Kropielnicka K, Bulińska K, et al. Insufficient modification of atherosclerosis risk factors in PAD patients. Adv Clin Exp Med 2018;27:819-26.

53. Slovut DP, Kargoli F, Fletcher JJ, et al. Quality of care among patients undergoing lower extremity revascularization. Vasc Med 2014;19:368-75

54. Steenhof N, Le Piane F, Leblanc K, et al. Vascular quality of care pilot study: how admission to a vascular surgery service affects evidence-based pharmacologic risk factor modification in patients with lower extremity peripheral arterial disease. Vasc Health Risk Manag 2014;10:333-40.

55. Thiney M, Della Schiava N, Ecochard R, et al. Effects on mortality and cardiovascular events of adherence to guideline-recommended therapy 4 years after lower extremity arterial revascularization. Ann Vasc Surg 2018;52:138-46. 
56. Willey J, Mentias A, Vaughan-Sarrazin M, et al. Epidemiology of lower extremity peripheral artery disease in veterans. J Vasc Surg 2018;68:527-35.e25.

57. Hasimu B, Li J, Yu J, et al. Evaluation of medical treatment for peripheral arterial disease in Chinese high-risk patients. Circ J 2007;71:95-9.

58. Conte MS, Bradbury AW, Kolh P, et al. Global vascular guidelines on the management of chronic limb-threatening ischemia. Eur J Vasc Endovasc Surg 2019;58(Suppl):S1-109.e33.

59. Norgren L, Hiatt WR, Dormandy JA, et al. Inter-society consensus for the management of peripheral arterial disease (TASC II). J Vasc Surg 2007;45(Suppl S):S5-67.
60. Li B, Salata K, de Mestral C, et al. Perceptions of Canadian vascular surgeons toward pharmacologic risk reduction in patients with peripheral artery disease: 2018 update. Ann Vasc Surg 2019; 58:166-73.e4.

61. Correa VC, Lugo-Agudelo LH, Aguirre-Acevedo DC, et al. Individual, health system, and contextual barriers and facilitators for the implementation of clinical practice guidelines: a systematic metareview. Health Res Policy Syst 2020;18:74.

62. Weitz JI, Angiolillo DJ, Geisler T, et al. Dual pathway inhibition for vascular protection in patients with atherosclerotic disease: rationale and review of the evidence. Thromb Haemost 2020; 120:1147-58. 